\title{
Urothelial carcinoma
}

\author{
Axel S. Merseburger $\cdot$ Shahrokh F. Shariat
}

Received: 16 June 2011 / Accepted: 14 July 2011 / Published online: 21 July 2011

(C) Springer-Verlag 2011

After a long period of smoldering activity in urothelial carcinoma research, interest this orphan disease has caught fire. This renewed enthusiasm has been fanned largely by a more sophisticated understanding of its biology, natural history, and application of novel technologies. Specifically, the realization that individual investigators are less likely to make an impact in this disease has led to highly prolific multi-institutional collaborations. In this special issue of the World Journal of Urology, we have compiled a series of outstanding articles on lower and upper tract urothelial carcinoma focusing on novel diagnostic approaches, insights into predictive factors, and innovations in surgical technology.

Bladder cancer represents an important cause of morbidity and mortality. In 2010, once again, it is the second most common genitourinary cancer in the United States, with a projected 70,530 new cases and 14,680 deaths [1]. Currently, it is estimated that there are more than 1,000,000 men and women alive in the United States and Europe who have a history of bladder cancer. At the time of initial diagnosis, approximately $70 \%$ of patients have cancers confined to the epithelium or the subepithelial connective tissue. These cancers usually are managed with endoscopic resection and the selective use of intravesical therapy. The recurrence rate for these tumors ranges from 50 to $70 \%$ and

A. S. Merseburger ( $\square)$

Department of Urology and Urologic Oncology,

Medical School of Hannover [MHH],

Carl Neuberg Str. 1, 30625 Hannover, Germany

e-mail: Merseburger.Axel@mh-hannover.de

S. F. Shariat

Weill Medical College of Cornell University,

525 East 68th Street, Starr 900, New York, NY 10065, USA between 10 and $15 \%$ progress to muscle-invasive disease over 5 years $[2,3]$. Disease recurrence may occur locally or in the upper urinary tract even after several years, necessitating lifelong surveillance. Management of this population is costly because of the extended surveillance and repeated use of endoscopic and intravesical therapies. Patients with muscle-invasive and metastatic disease have a much more precarious survival outcome and also contribute greatly to the cost of bladder cancer care because of the expense of radical cystectomy and systemic chemotherapy. As a result, bladder cancer is the most expensive cancer in the United States [4].

To help refine the management of non-muscle-invasive bladder cancer, Hernándes and coworkers have validated the EORTC risk tables concluding that the tool initially introduced and published by Sylvester et al. accurately predicts the recurrence of the patients. In this retrospective evaluation, based on a prospective cohort of 417 patients following resection of TaT1 bladder malignancies, recurrence and progression rates were as predicted by the EORTC risk tables (53.46 and $8.43 \%$ at 5 years). However, due to the sample size, only recurrence was statistically validated and larger cohorts should clarify the accuracy of those valuable tables.

There is still controversy about the optimal management of high-grade non-muscle-invasive bladder urothelial cancer. Both the American Urological Association and European Association of Urology recommend initial intravesical treatment with bacillus Calmette-Guerin (BCG) followed by maintenance therapy. While the complete response rate to BCG therapy in patients with high-risk NMIBC can be high, most patients experience eventually disease recurrence. In an excellent review, Yates and Rouprêt discuss the management of patients who experience failure of intravesical BCG therapy. First, they characterize the different 
types of BCG failure (i.e., BCG-intolerance, BCG-resistance, BCG-relapsing, and BCG-refractory disease), and then, they critically review the literature on radical cystectomy and bladder-sparing treatment options other than radiation therapy. They discuss the efficacy of treatment options such as intravesical chemotherapy or immunotherapy, deviceassisted therapeutic modalities, and combination therapies. This review underlines the strong need for further research to help identify which of these therapies is the most appropriate and to develop evidence-based guidelines. Until then, in our opinion, all conservative treatments should be considered investigational and radical cystectomy remains the standard of care for high-risk patients who have failed BCG therapy.

There is no doubt that novel technologies are needed to make change the outcomes of patients with urothelial cancer. Transurethral resection of bladder tumors using electrical current which is used to diagnose, stage, and treat (some) bladder cancers results in thermal damage and possible fragmentation and suspension of urothelial carcinoma cells, which are thought to be responsible for a significant number of tumor recurrences. Nagele and associates have therefore tested in a pilot study of five patients the performance of water jet hydrodissection for removing papillary non-muscle-invasive bladder cancer. This technology holds the promise of removing superficial tumors while preserving its histological structures. They found that the technique was feasible and pathologist could assess the entire lamina propria and the resection edges due to the en bloc resection. These promising findings require confirmation in larger well-controlled trials, which are underway. Similarly, Wolters and colleagues have tested a Thulium:Yag laser in six patients to demonstrate feasibility of en bloc mucosectomy for accurate staging of bladder cancer. They found that the technique was safe and effective treatment. Oncologic outcomes were also assessed. This and other lasers are awaiting broader testing and will eventually enter our armamentarium for the management of urothelial carcinomas as excellently been described in the review of Kramer.

Park and colleges have evaluated an interesting topic if the number of lymph nodes demonstrating in a multivariate analysis that $\mathrm{T}$ stage and lymphovascular invasion were significant predictors for survival in N0 patients, whereas adjuvant chemotherapy and LN density were predictors for survival in $\mathrm{N}+$ patients. However, in this study, the number of excised lymph nodes was not associated with improved prognosis. There is a German ongoing prospective multicenter trial (LEA trial) that will deliver further insights expected to be published at the end of 2011.

Gakis and coworkers have looked at the accuracy of frozen section analysis for detecting and eliminating malignant ureteral margins at radical cystectomy demonstrating a high specificity and reliability to detect malignant remaining disease. Frozen section results were false positive in 1/402 margins, demonstrating a reliable pathologic intraoperative result (specificity: $99.8 \%$ ). Patients with positive final margins are at increased risk of UUT recurrences.

Upper tract urothelial carcinoma is an uncommon disease with little evidence-based data to guide clinical decision making. Within the last 3 years, the tools available for the diagnosis and management of upper tract urothelial carcinoma have improved significantly, complementing a growing understanding of the biology of this disease. Upper tract urothelial carcinoma requires appropriate management at all stages, since both the cure rate and morbidity are very sensitive to nuances of treatment. Yet, proper risk stratification remains a challenge owing to the difficulty of clinical staging. A comprehensive review and four welldone studies address clinical challenges and the value of various prognostic markers in the management of this disease.

The role of lymph node dissection in patients treated with radical nephroureterectomy for upper tract urothelial cancer is still controversial. Regional nodes are frequently involved by upper tract urothelial cancer and represent the most common metastatic site. Nodal status is a significant predictor of patient outcomes, especially in invasive disease. A previous study has shown that $\mathrm{pNx}$ status is associated with a worse prognosis than pN0 in pT2-4 tumors [5]. To test the value of lymph node dissection in upper tract urothelial carcinoma, Burger and coworkers collected a large data set from nine international centers. They confirmed that while performing a lymphadenectomy does not impact clinical outcomes in patients with localized upper tract urothelial cancer, in locally advanced upper tract urothelial cancer, lymphadenectomy allows for improved staging and survival. Taking the results of this and other studies together, one can conclude that in the absence of prospective trials, patients expected to have pT2-4 upper tract urothelial carcinoma should undergo a thorough lymphadenectomy to improve staging and thereby help guide decision making regarding adjuvant therapy and risk-adjusted follow-up scheduling. Similar to other cancers, it is likely that radical surgery and lymphadenectomy might be curative in a subpopulation of upper tract urothelial cancer patients with limited nodal disease. The task is now to identify the limits and template of lymphadenectomy for upper tract urothelial cancer.

Chromecki et al. report that higher chronological age confers worse prognosis in patients with upper tract urothelial carcinoma in a study of 1,169 patients treated with radical nephroureterectomy. They hypothesize that the worse oncologic outcomes in elderly patients treated with radical nephroureterectomy could be due to biological factors (i.e., tumor aggressiveness and host defence) and/or suboptimal 
quality of care. However, they also found that a large proportion of elderly patients were cured with radical nephroureterectomy suggesting that chronological age alone is an inadequate indicator criterion to predict response of older patients to radical nephroureterectomy. This study underlines that before denying an elderly patient radical surgery, the benefits of local disease control conferred with surgery must be weighed against the potential perioperative mortality and morbidity. Further work is needed to improve our understanding of the reasons for worse cancer-specific outcomes in the elderly and to develop evidence-based strategies to improve cancer care in this growing segment of the population.

Shariat et al. found that women were less likely to have high-grade disease, undergo lymph node dissection, and to receive adjuvant chemotherapy. Despite these differences between genders, this study confirms previous studies showing that there is no difference in outcomes between men and women treated with radical nephroureterectomy for upper tract urothelial carcinoma [6, 7]. With regard to the lower urinary tract, Fajkovic has summarized the most recent findings on this topic in an excellent review with literature search going back more than 35 years. Recent population-based and multicenter studies have demonstrated that female gender is associated with a significant higher rate of cancer-specific recurrence and mortality after radical cystectomy. The future should focus on tailored gender-specific treatment and research for bladder cancer patients.

Otto et al. investigated the prognostic value of concomitant carcinoma in situ in upper tract urothelial carcinoma in a large, international cohort of patients treated with radical nephroureterectomy. They found that the prevalence of concomitant carcinoma in situ of the upper urinary tract increased with worsening cancer features such as advancing pathologic stage, the presence of lymph node metastasis, and the presence of lymphovascular invasion. More importantly, the authors found that in patients with organconfined upper tract urothelial carcinoma, those with concomitant carcinoma in situ were at significantly increased risk of both disease recurrence and cancer-related mortality. The authors rightfully concluded that the presence of concomitant carcinoma in situ should be included in the reports of radical nephroureterectomy specimens and that in patients with organ-confined upper tract urothelial carcinoma; concomitant carcinoma in situ should be included in predictive tools for clinical decision-making regarding therapy and follow-up.
Finally, in a comprehensive review article, Verhoest et al. discuss the clinical value of prognostic and predictive markers in the management of upper tract urothelial carcinoma. This article represents a state of the art update on a field that is rapidly evolving. While most clinical decision making in this disease was until recently based on extrapolation from lower tract urothelial carcinoma, a revolution in research activity in this field has resulted in much progress in our understanding of upper tract urothelial carcinoma and is paving the way for evidence-based management of this disease.

We hope you will enjoy reading this special edition as much as we did compiling it for you.

Sincerely yours,

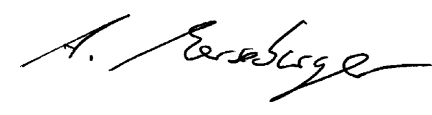

Axel S. Merseburger

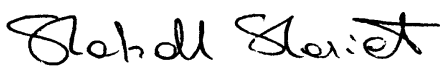

Shahrokh F. Shariat

\section{References}

1. Jemal A, Siegel R, Xu J et al (2010) Cancer Statistics. CA Cancer J Clin 60(5):277-300

2. Prout GR, Barton BA, Griffin PP et al (1992) Treated history of noninvasive grade 1 transitional cell carcinoma. The National Bladder Cancer Group. J Urol 148:1413-1419

3. Pagano F, Bassi P, Galetti TP et al (1991) Results of contemporary radical cystectomy for invasive bladder cancer: a clinicopathological study with an emphasis on the inadequacy of the tumor, nodes and metastases classification. J Urol 145:45-50

4. Botteman MF, Pashos CL, Redaelli A et al (2003) The health economics of bladder cancer: a comprehensive review of the published literature. Pharmacoeconomics 21:1315-1330

5. Roscigno M, Shariat S, Margulis V et al (2009) Impact of lymph node dissection on cancer specific survival in patients with upper tract urothelial carcinoma treated with radical nephroureterectomy. J Urol 181:2482-2489

6. Lughezzani G, Sun M, Perrotte P et al (2010) Gender-related differences in patients with stage I to III upper tract urothelial carcinoma: results from the surveillance, epidemiology, and end results database. Urology 75:321-327

7. Fernández M, Shariat S, Margulis V et al (2009) Evidence-based sex-related outcomes after radical nephroureterectomy for upper tract urothelial carcinoma: results of large multicenter study. Urology $73: 142-146$ 